\title{
Bauhinia bauhinioides Kallikrein Inhibitor Fragments with Bradykinin-Like Activities
}

\author{
Flavio A. Lopes ${ }^{1}$, Jones M. Silva ${ }^{1}$, Maria Luiza V. Oliva ${ }^{2}$, and \\ Antonio Miranda ${ }^{1}$ \\ ${ }^{I}$ Department of Biophysics and ${ }^{2}$ Department of Biochemistry, Federal University of São Paulo, \\ São Paulo, SP, 04044-020, Brazil
}

\section{Introduction}

Medicinal plants extracts contain many proteinase inhibitors that could be an interesting source of new drugs. Structural modifications in some of this kind of macromolecule could also lead to compounds with interesting biological properties [1]. In this work, we studied one peptide fragment (BbKI $\left.\mathrm{B}_{51-62}\right)$ of Bauhinia bauhinioides kallikrein inhibitor $(\mathrm{BbKI})[2,3]$ and its analogue $\left(\mathrm{P}_{2}\right)$ that was designed by the insertion of a proline residue in order to mimic the $N$-terminal region of the bradykinin (BK) molecule. Both retro inverso counterparts $\mathrm{Ri}-\mathrm{BbKI}_{51-62}$ and $\mathrm{Ri}-\mathrm{P}_{2}$ were also included. We evaluated the capability of the peptides to induce contraction on stomach fundus of mouse as well as their capability to induce calcium release from guinea pig ileum cell culture. Both biological effects induced by the fragments were compared with the ones caused by BK.

\section{Results and Discussion}

Peptides (Table 1) were manually synthesized by using the Boc-strategy [4]. After RP-HPLC purification, they were characterized by LC-ESI-MS. Stomach fundus was isolated from mouse and mounted into organ bath containing modified Krebs-Ringer solution at $37^{\circ} \mathrm{C}(\mathrm{pH} 7.4)$. The maximal effect was obtained from comparison of contractile responses induced by carbachol $10^{-6} \mathrm{M}$ (Figure 1A). Calcium release was determined by Fluo-4 dye. Fluorescence was determined by a FlexStation 3 System (Molecular Devices) (Figure 1B). Peptides resistance to degradation by enzymes presents on human blood were also evaluated (data not shown). Conformational studies by Circular Dichroism in different environments were performed (data not shown). The full results of this study is presented elsewhere [5].

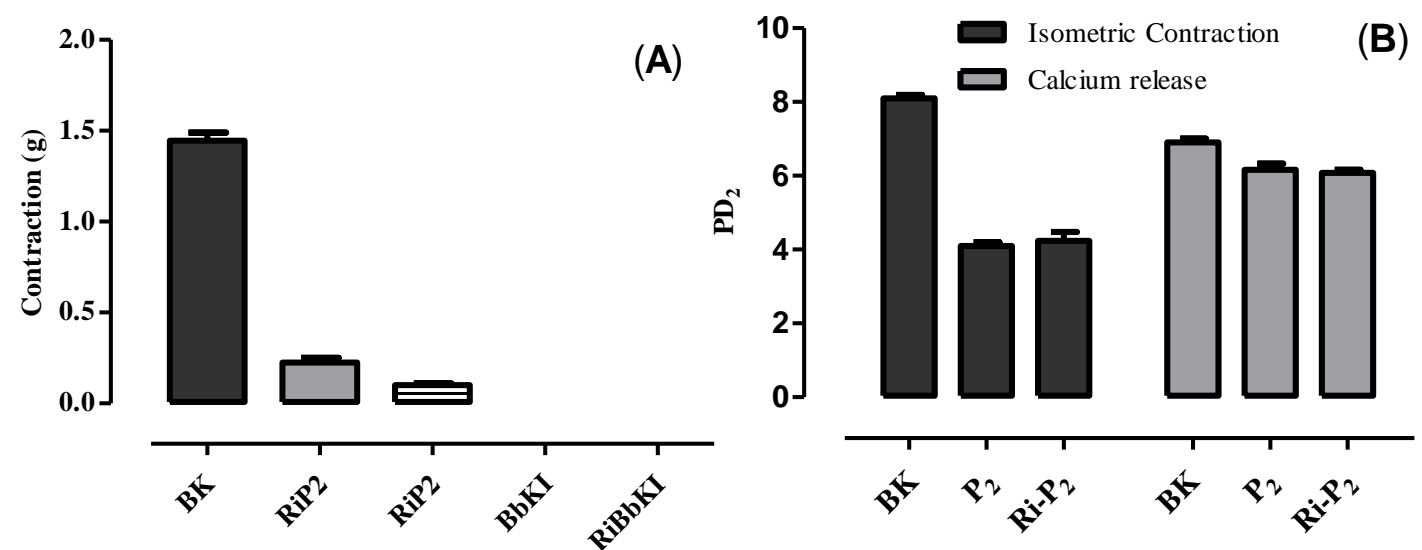

Fig. 1. Isometric contraction induced by $B K$ and BbKI related fragments with maximum contraction at $10^{-5} \mathrm{M}$ concentration $(\mathrm{A})$ and their corresponding $\mathrm{pD}_{2}$ values for isometric contraction and calcium release $(B)$. 
Table 1. BbKI related peptide fragments studied.

\begin{tabular}{cc}
\hline Name & Sequence $^{a}$ \\
\hline BK & R-P-P-G-F-S-P-F-R \\
BbKI $_{51-62}$ & R-P-G-L-P-V-R-F-E-S-P-L-NH 2 \\
Ri-BbKI $_{51-62}$ & 1-p-s-e-f-r-v-p-l-g-p-r-NH \\
$\mathrm{P}_{2}$ & R-P-P-G-L-P-V-R-S-F-S-P-L-R-NH \\
Ri-P $_{2}$ & r-l-p-s-f-s-r-v-p-l-g-p-p-r-NH \\
\hline
\end{tabular}

${ }^{a}$ small letters correspond to D-amino acids.

Neither BbKI $_{51-62}$ nor Ri-BbKI ${ }_{51-62}$ presented bradykinin-like activity, but as expected, the retro-inverso compound was resistant to the enzymes presented in the blood plasma. $\mathrm{P}_{2}$ and Ri- $\mathrm{P}_{2}$ were able to induce a contractile response on gastric smooth muscle, maybe acting via B2 BK-receptor activation. $\mathrm{P}_{2}$ and Ri- $\mathrm{P}_{2}$ also produce calcium release from Guinea pig ileum muscle cells in a similar way than BK. Circular Dichroism conformational studies did not show a direct correlation between the biological effects and the peptides structure since all compounds presented a CD profile of disordered conformations.

\section{Acknowledgments}

This work was supported by grants and fellowships from CNPq and FAPESP.

\section{References}

1. Oliva, M.L.V., et al. Current Protein \& Peptide Science 12, 348-357 (2011), http://dx.doi.org/10.2174/138920311796391061

2. Oliva, M.L.V., et al. Immunopharmacology 45, 163-169 (1999), http://dx.doi.org/10.1016/S0162-

3109(99)00075-2

3. Andrade, S.S., et al. Biological Chemistry 393, 943-957 (2012), http://dx.doi.org/ 10.1515/hsz-2012-0126

4. Fazio, M.A., et al. Biopolymers 84, 205-218 (2006), http://dx.doi.org/10.1002/bip.20396

5. Alves, F.L., et al. J. Peptide Science 21, $495-500$ (2015), http://dx.doi.org/10.1002/psc.2766 